\title{
Epidemiology of Posttransplantation Lymphoproliferative Disorder in Adult Renal Transplant Recipients
}

\author{
Muir Morton, ${ }^{1}$ Beatrice Coupes, ${ }^{1}$ Stephen A. Roberts, ${ }^{2}$ Paul E. Klapper, ${ }^{3,5}$ Richard J. Byers, ${ }^{4}$ \\ Pamela J. Vallely, ${ }^{5}$ Kate Ryan, ${ }^{6}$ and Michael L. Picton ${ }^{1}$
}

\begin{abstract}
Background. There is little information in the literature describing the relationship between posttransplantation lymphoproliferative disorder (PTLD) incidence and presentation with both recipient Epstein-Barr virus (EBV) serostatus and EBV status of PTLD histology, particularly in the late posttransplantation period.

Methods. This study reports the largest UK single-center, single-organ analysis of PTLD to date in a retrospective cohort study of 80 cases occurring in 4189 adult renal transplant recipients.

Results. The incidence rate was 2.6 cases per 1000 patient-years (95\% confidence interval [95\% CI], 2.1-3.2) for PTLD, 1.8 (95\% CI, 1.4-2.4) for non-Hodgkin's lymphoma, and 0.2 (95\% CI, 0.07-4.2) for Hodgkin's lymphoma. NonHodgkin's lymphoma occurred at a rate 7.6 times that of the adult general population in England, whereas the rate for Hodgkin's lymphoma was 5.9 times. The incidence of PTLD was highest during the 10th to 14th posttransplantation years. Early-onset disease was associated with EBV-seronegative recipient status, EBV-positive histology, and the involvement of extranodal sites. PTLD occurring in EBV-seronegative recipients was associated with EBV nuclear antigen antibody deficiency, polymorphic disease, and the involvement of extranodal sites. EBV-negative histology occurred in $32 \%$ of cases at a median time to presentation of 109 months. PTLD involving the allograft, central nervous system, and skin was uncommon and occurred late.

Conclusion. The incidence of PTLD is highest in the late posttransplantation period. Close clinical surveillance and education for transplant recipients is required for the duration of time while immunosuppressed. Failure to detect EBV DNA in blood should not reassure, particularly in patients with symptoms such as abdominal pain, oropharyngeal complaints, neck lumps, and B-symptoms.
\end{abstract}

Keywords: Epstein-Barr virus, PTLD, Renal transplantation.

(Transplantation 2013;95: 470-478)

$\mathrm{P}$

osttransplantation lymphoproliferative disorder (PTLD) is the second most common malignancy after skin cancer occurring in solid organ transplant recipients (1). It occurs in $1 \%$ to $3 \%$ adult renal transplant recipients and is associated with poor survival rates after diagnosis (2-5). Implicated in the disease pathogenesis is exposure to immunosuppression

Funding support for the study was provided by the Central Manchester University Hospitals Foundation Trust Biomedical Research Centre in the form of research grants awarded to the team in open competition. M.M. received an Astellas Renal Transplant Clinical Fellowship, and M.L.P. received an unrestricted educational grant from Amgen.

The authors declare no conflicts of interest.

${ }^{1}$ Department of Renal Medicine, Central Manchester University Hospitals Foundation Trust, Manchester, UK.

${ }^{2}$ Health Sciences Methodology, University of Manchester, Manchester, UK.

${ }^{3}$ Department of Clinical Virology, Central Manchester University Hospitals Foundation Trust, Manchester, UK.

${ }^{4}$ Department of Histopathology, Central Manchester University Hospitals Foundation Trust, School of Cancer and Enabling Sciences, Faculty of Medical and Human Sciences, University of Manchester, and Manchester Academic Health Sciences Centre, Manchester, UK.

5 Microbiology and Virology Unit, School of Translational Medicine, University of Manchester, Manchester Academic Health Sciences Centre, Manchester, UK.
$(4,6)$, impairment of cellular immunity, and the infective agent Epstein-Barr virus (EBV) (7-11). PTLD tissue is EBV positive in up to $75 \%$ of cases (12), and EBV-seronegative recipients at risk of primary infection have a markedly increased risk of disease (12-15). Recent PTLD registry reports for kidney transplant recipients have shown bimodal patterns of incidence, with peaks in the first year and then in the later posttransplantation period $(6,12,14,15)$.

\footnotetext{
${ }^{6}$ Department of Haematology, Central Manchester University Hospitals Foundation Trust, Manchester, UK.

Address correspondence to: Dr. Muir Morton, M.B.B.S., M.R.C.P.(UK), M.A.Hons., Department of Renal Medicine, Central Manchester University Hospitals Foundation Trust, Oxford Road, Manchester M13 9WL, UK. E-mail: muir.morton@cmft.nhs.uk

M.M., B.C., S.A.R., P.E.K., P.J.V., K.R., and M.L.P. participated in the research design. M.M., B.C., P.E.K., R.J.B., P.J.V., K.R., and M.L.P. participated in the writing of the article. M.M., B.C., P.E.K., R.J.B., and K.R. participated in the performance of the research. M.M. and S.A.R. participated in the data analysis.

Received 24 August 2012. Revision requested 11 September 2012.

Accepted 27 September 2012.

Copyright (C) 2012 by Lippincott Williams \& Wilkins

ISSN: 0041-1337/13/9503-470

DOI: 10.1097/TP.0b013e318276a237
} 
There is little information in the literature describing relationships between PTLD incidence and presentation with both recipient EBV serostatus and EBV status of histology, particularly in the late posttransplantation period. Focus on late PTLD in adult kidney transplant recipients is increasingly important as median graft and patient survival times increase (now $>80 \%$ at 5 years).

To address the points above, we performed an observational cohort study of our renal transplant population to identify PTLD cases, define incidence rates of PTLD, and describe the variation in clinical presentation, including organ involvement. We analyzed the timing of disease presentation with reference to EBV recipient serostatus and tissue EBV status, comparing those patients with early ( $<1$ year), late $(>1$ year), and very late $(>10$ years $)$ onset of disease.

\section{RESULTS}

\section{Incidence of Posttransplantation Lymphoproliferative Disorder}

A summary of the incidence data is shown in Table 1.

The median follow-up time for all transplant recipients was 2058 days ( 5.6 years; interquartile range [IQR], 676-3983). PTLD occurred in 80 recipients in 30,867 patient-years, giving an incidence rate of 2.6 cases per 1000 patient-years. NonHodgkin's lymphoma (NHL) was seen more frequently than Hodgkin's lymphoma (rate ratio, 9.7). NHL occurred at a rate ratio of 7.6 and Hodgkin's lymphoma at 5.9 that of the general population (16). PTLD occurred more frequently in males than females $(P=0.039)$ and there was a trend toward increased rates with age at transplantation. No significant differences in incidence rates were detected between different eras of transplantation (Tables 1 and 2).

Incidence rates were associated with time from transplantation. The second to fourth years after transplantation were used as a reference group because this period was expected to have the lowest incidence of PTLD. We observed a bimodal pattern of presentation with rates in the first year after transplantation higher than the reference period (rate ratio, 2.7; 95\% confidence interval [95\% CI], $1.1-6.1 ; P=0.0098)$ and peak incidence occurring later between 10 and 14 years after transplantation (rate ratio, 3.4; 95\% CI, 1.7-7.3; $P=0.032$; Fig. 1).

\section{Posttransplantation Lymphoproliferative Disorder Clinical Characteristics}

The characteristics of the PTLD population are summarized in Table 3.

The median time from transplantation to presentation of PTLD for the 80 cases was 74 months 6.1 years; IQR, 28-131). Cases presented early (within 1 year after transplantation) in 12 of $80(15 \%)$ and late (>1 year) in 68 of $80(85 \%)$. Very late presentations ( $>10$ years) occurred in 24 of $80(30 \%)$, with $3(4 \%)$ beyond the 20th posttransplantation year.

\section{Early-Onset Disease}

Early-onset disease ( $<1$ year) was associated with EBVpositive tissue status (odds ratio [OR], 15; 95\% CI, 1.8-128; $P=0.005$ ), viral capsid antigen (VCA) positive, EBV nuclear antigen (EBNA) antibody-negative status (OR, 10.7; 95\% CI, 1.1-100.5; $P=0.02$ ), prior anti-thymocyte globulin (ATG) therapy (OR, 4.8; 95\% CI, 1.1-20.6; $P=0.048$ ), extranodal involvement (OR, 7.0; 95\% CI, 0.8-57.3; $P=0.0497$ ), Ann Arbor staging 3 or more (OR, 4.5; 95\% CI, 1.2-16.9; $P=0.02$ ), diseases involving the liver (OR, 5.6; 95\% CI, 1.4-22.6; $P=0.02$ ) and the spleen (OR, 14.5; 95\% CI, 3.1-66.2; $P=0.0008)$, and gastrointestinal tract involvement (OR, 3.7; 95\% CI, 0.9-14.2; $P=0.059)$.

\section{Late-Onset Disease}

Late-onset ( $>1$ year) disease involved extranodal sites in $62 \%$ of cases and was EBV tissue-positive PTLD in 29 of 46 $(63 \%)$. All cases of disease involving the graft, central nervous system (CNS), skin, soft tissue, pericardium, and 5 of 6 cases with bone marrow involvement in our series occurred late.

\section{Very Late Disease}

PTLD occurred very late ( $>10$ years) in 24 individuals. Relapses were seen in $6(25 \%)$ of these very late cases compared with 5 of $44(11 \%)$ cases presenting between 1 and 10 years and 0 in early-onset disease. Sites of disease included the neck $(n=10)$, oropharynx $(n=5)$, two at sites of chronic inflammation including a tooth abscess and hyperplastic gum, groin nodes $(n=5)$, gastrointestinal tract $(n=2)$, allograft $(\mathrm{n}=1)$, and CNS $(\mathrm{n}=2)$. Histology was negative for EBV in 5 of $21(24 \%)$, and 4 of $19(24 \%)$ of these cases occurred in seronegative recipients.

\section{Pretransplantation Serostatus and Time to Presentation}

Pretransplantation EBV serostatus was obtained for $70 \%$ of the PTLD cases, of which 19 of 56 (34\%) were seronegative and 37 of $56(66 \%)$ were seropositive. The median time to PTLD for seronegative recipients was shorter than for seropositive (19 months [IQR, 7-80] vs. 115 months [IQR, 54-140]; $P=0.003$; Fig. 2A), and nearly half of all cases in the seronegative group occurred during the first transplantation year. Early-onset disease occurred almost entirely in seronegative recipients (90\% cases with known serostatus; OR, 32.4 for seronegative versus seropositive; 95\% CI, 3.7-287; $P<0.0001$; Fig. 2A). PTLD presenting early in seropositive recipients was unusual with only a single case in the first year and two cases during the second year, both early-type lesions.

PTLD in seronegative recipients was associated with VCA-positive EBNA antibody deficiency (OR, 23.4; 95\% CI, 24.0-161; $P \leq 0.0001)$, early onset $(P=0.003)$, polymorphic histology (OR, 5.2; 95\% CI, 1.1-24; $P=0.049$ ), extranodal involvement (OR, 4.7; 95\% CI, 1.2-19.3; $P=0.036$ ), Ann Arbor staging 3 or more $(\mathrm{OR}, 3.9 ; 95 \% \mathrm{CI}, 1.2-13.0 ; P=0.04)$, and disease involving the liver (OR, 3.9; 95\% CI, 0.9-15.4; $P=0.08$ ) and spleen (OR, 5.3; 95\% CI, 1.2-23.7; $P=0.030$ ).

\section{Epstein-Barr Virus-Negative Histology Posttransplantation Lymphoproliferative Disorder}

EBV status of PTLD histology was available for 56 of 80 cases, of which 18 of 56 (32\%) were EBV negative. EBVnegative PTLD was unusual in the early posttransplantation period and case numbers increased with time from transplantation: 1 of $11(9 \%)$ in the first year, 3 of $8(38 \%)$ in the second to fourth years, 6 of $12(50 \%)$ in the fifth to ninth years, and 9 of 26 (35\%) cases beyond 10 years (Fig. 2B). EBV was detectable in blood at diagnosis in 55\% of EBV-negative histology disease. EBV-negative PTLD was seen in $24 \%$ of seronegative recipients and $37 \%$ of seropositive recipients 


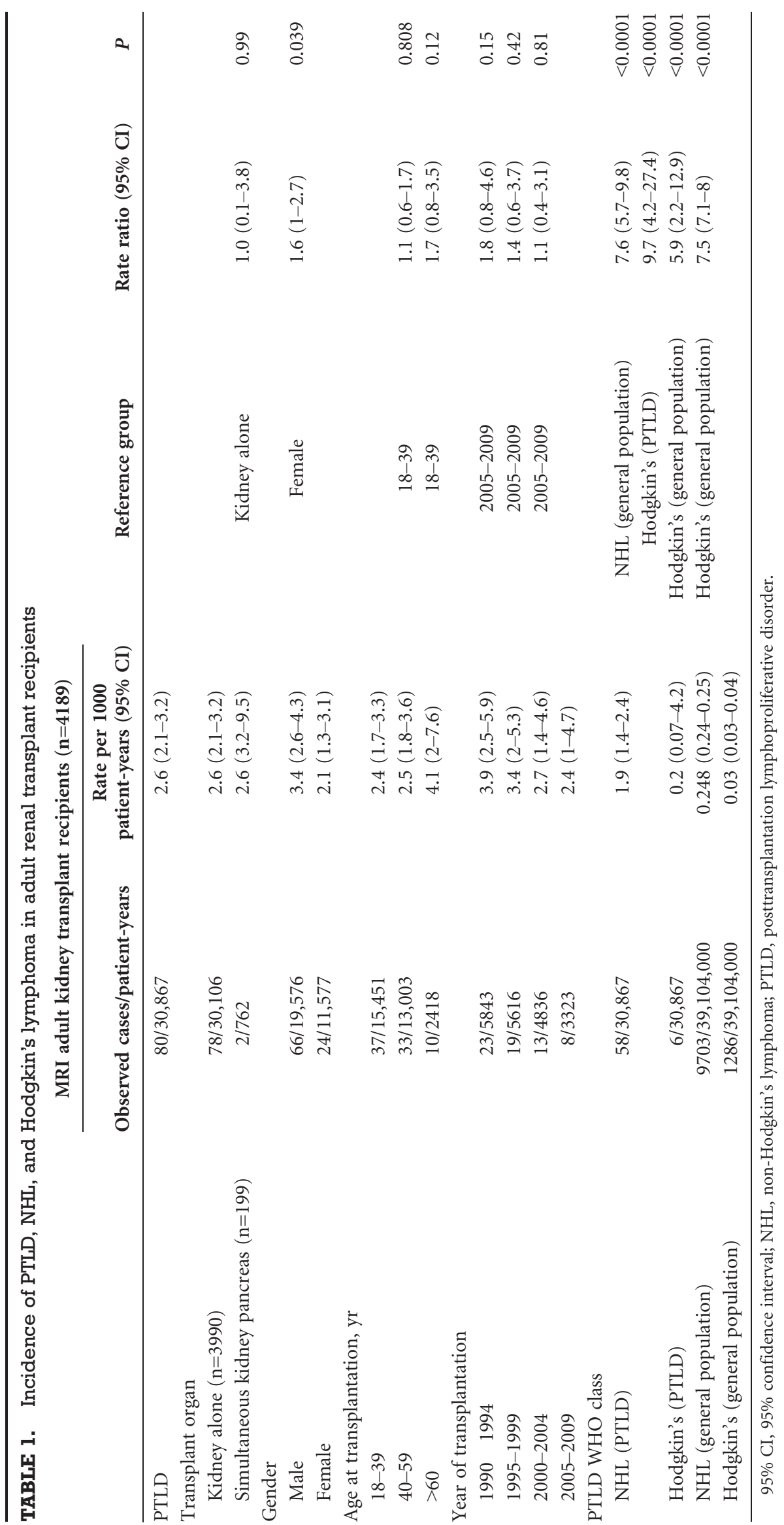


$(P=0.51)$. Seronegative recipients who developed EBV-negative PTLD all had evidence of seroconversion (detectable IgG to EBV VCA) at diagnosis.

\section{Epstein-Barr Virus-Positive Histology Posttransplantation Lymphoproliferative Disorder}

PTLD tissue was EBV positive in 38 of $56(68 \%)$ of cases, including 91\% (10 of 11) of all cases presenting within 1 year of transplantation. Detectable EBV viral loads in blood were seen in $96 \%$ of cases. The median time to presentation for patients with EBV-positive PTLD was slightly but not significantly earlier than EBV negative ( 89 vs. 109 months).

\section{Presenting Symptoms and Organ Involvement at Presentation}

The demographics, histology, presenting clinical symptoms, and sites of disease at the time of diagnosis are shown in Table 3. Symptoms occurring with the greatest frequency included neck swelling, abdominal pain, oropharyngeal complaints, and occurrence of B-symptoms.

\section{Survival}

Overall patient survival at 1,5 , and 10 years was $66 \%, 50 \%$, and $37 \%$, respectively, with a median survival of 1253 days from diagnosis. A complete response to treatment was seen in 46 of 80 (58\%) of patients, of whom 11 (24\%) subsequently relapsed at a median time from diagnosis of 325 days (IQR, 214-726) and 8 (17\%) lost their graft at a median time of 896 days (IQR, 363-1370) from diagnosis.

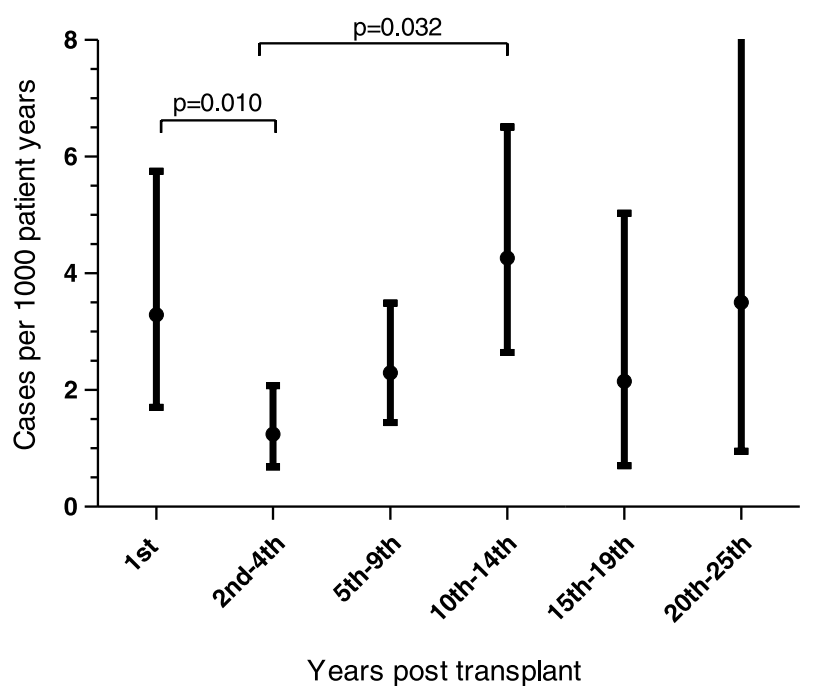

FIGURE 1. PTLD incidence rate versus time from transplantation for patients transplanted from 1968 to 2010. Incidence rates are presented as cases per 1000 patient-years with Poisson 95\% CI. Individuals were censored at diagnosis of PTLD, graft failure, death with a functioning graft, or on reaching the end of the follow-up period. 95\% CI, 95\% confidence interval; PTLD, posttransplantation lymphoproliferative disorder. 


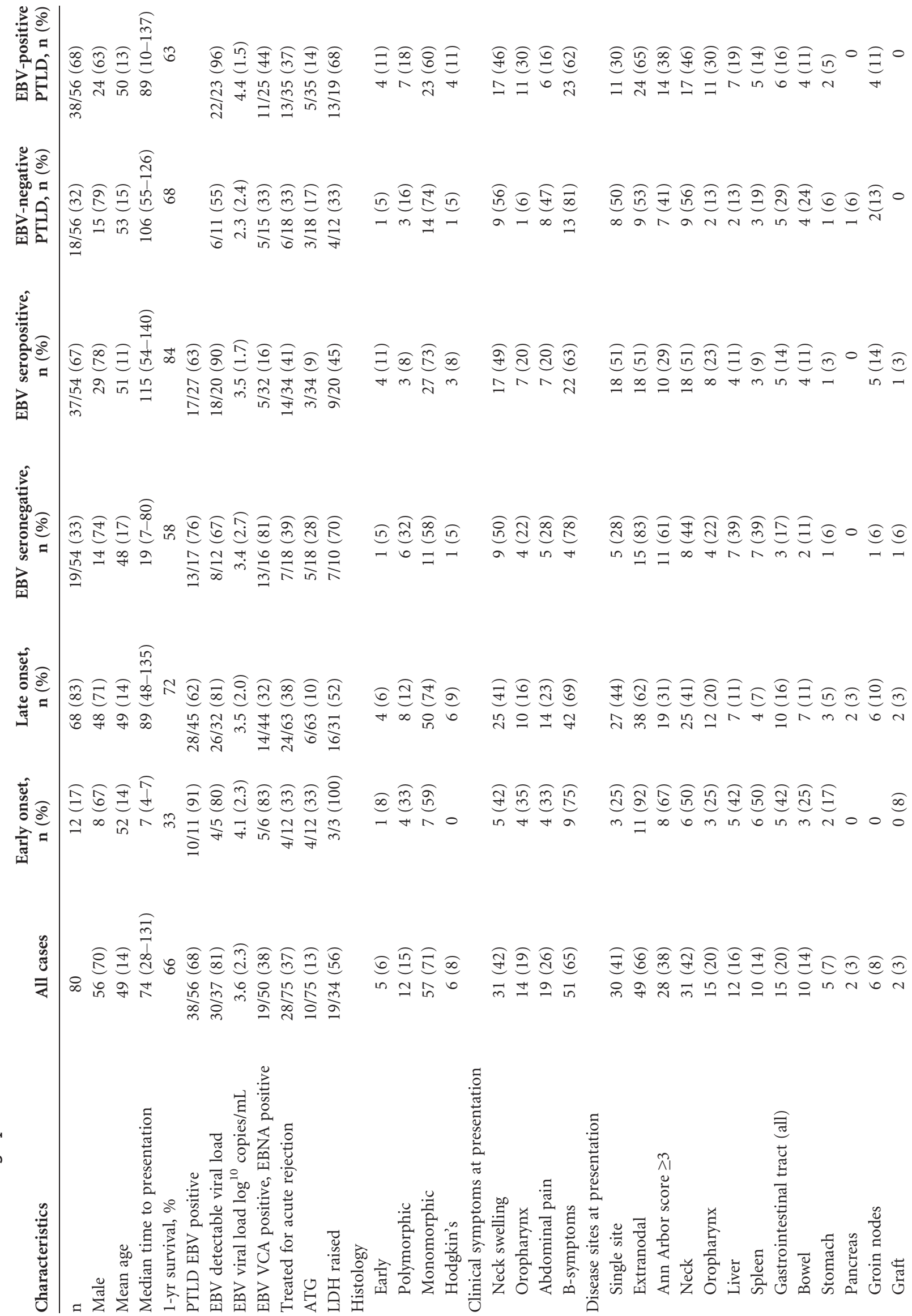




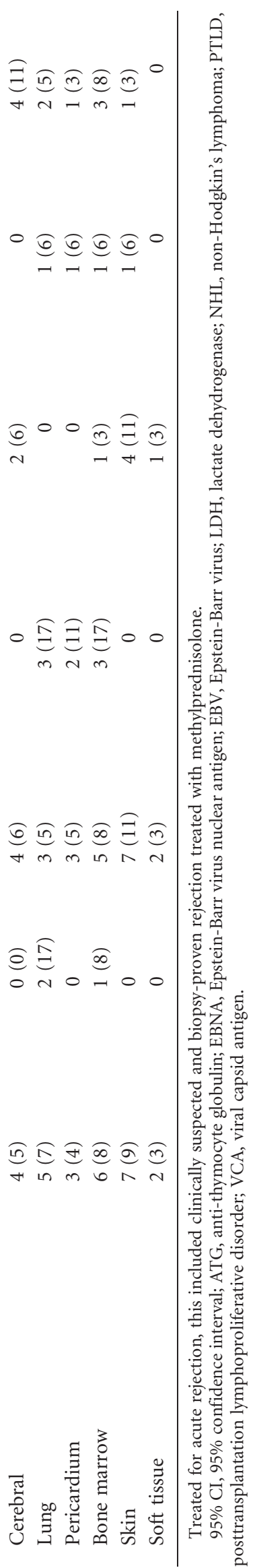

Graft survival at 1, 5, and 10 years from diagnosis in those who had a complete response was $96 \%, 82 \%$, and $77 \%$, respectively.

\section{DISCUSSION}

This study reports the largest UK single-center, singleorgan analysis of PTLD to date. PTLD presented up to 24 years after transplantation in $1.9 \%$ of adult kidney transplant recipients. Our incidence rates were similar to those reported recently by Swedish (6), French (12), US (15), and UK (5) studies. Analysis of PTLD timing in relation to EBV serostatus confirmed the increased risk for seronegative recipients in the first year but also highlighted a persistently increased risk for these patients in the late posttransplantation period. This study also draws attention to differences in clinical presentation for disease occurring early, late, and very late in EBVseronegative and EBV-seropositive recipients and for EBV histology-positive and histology-negative PTLD.

Compared with the adult general population, we found an eightfold increased risk of NHL and sixfold increased risk of Hodgkin's lymphoma. EBV positivity was found in approximately $40 \%$ of Hodgkin's lymphoma cases (17) and $26 \%$ of B-cell NHL cases (18) in the general population. In our series, EBV positivity was more prevalent and seen in $80 \%$ of early lesions, $80 \%$ of Hodgkin's lymphoma, $70 \%$ of polymorphic cases, and $64 \%$ of monomorphic PTLD cases.

High rates of lymphoma in solid organ transplant recipients are linked to the use of specific immunosuppressive agents, including calcineurin inhibitors $(4,5)$, and T-cell-depleting antibodies $(2,14,15)$. Caillard et al. observed a fall in lymphoma incidence more recently in France, which may have been due to reduced use of T-cell-depleting antibodies. We did not observe any statistically significant changes in recent times in our series, although we have never used T-cell-depleting agents as induction therapy. Compared with the French registry (2012) (12), a similar proportion of PTLD cases in our series involved gastrointestinal tract disease. We experienced less early-onset disease (15\% vs. $48 \%$ ), CNS disease (5\% vs. 20\%), and graft involving disease than the French series (3\% vs. 24\%). Reasons for these differences may be due to a low French rate of recipient-seronegative status at transplantation (only 3\%), with only $16 \%$ of French cases occurring in seronegative recipients compared with $34 \%$ in our series. Further, the French had a high rate of ATG induction, 54\% in controls and 66\% in cases, compared with only $13 \%$ of our cases.

Our data confirm the first year after transplantation as a period of high incidence of PTLD for EBV-seronegative recipients $(12,13,15)$. French and US registry data have also associated EBV-seronegative status with an increased hazard risk of not only early but also late PTLD. In our series, 90\% of early and a further $22 \%$ of all late PTLD occurred in seronegative recipients despite them making up less than $10 \%$ of all adult kidney transplant recipients in our unit. The small number of seronegative recipients and relatively high proportion of cases presenting in this group in the first year and later ( $>50 \%$ of cases) suggests that these individuals should remain under close clinical surveillance for the duration of their transplant life. Disease occurring in our seropositive recipients was unusual in the first few years. Seropositive recipients had a significantly greater likelihood of PTLD in the late follow-up 
A

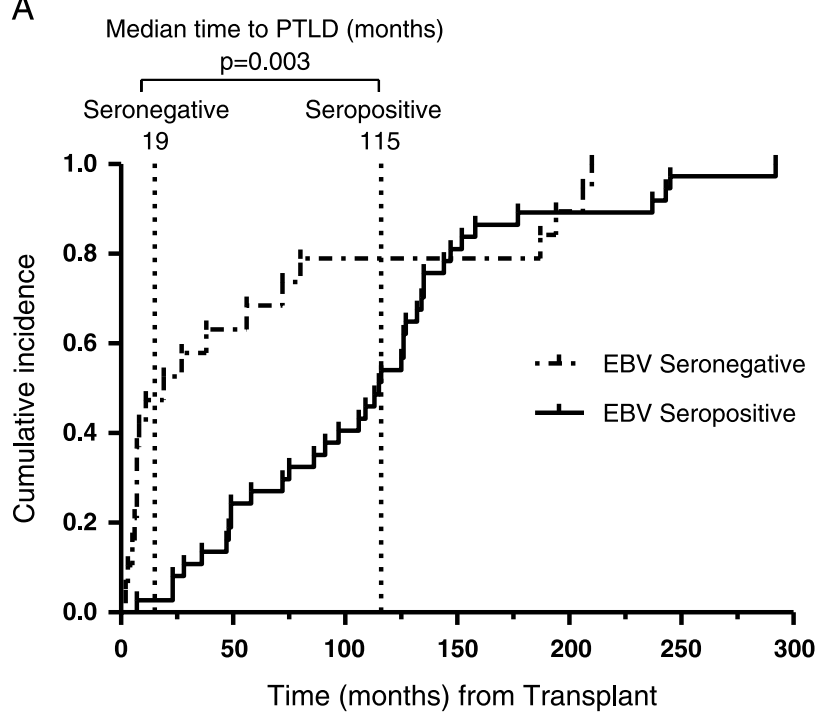

B

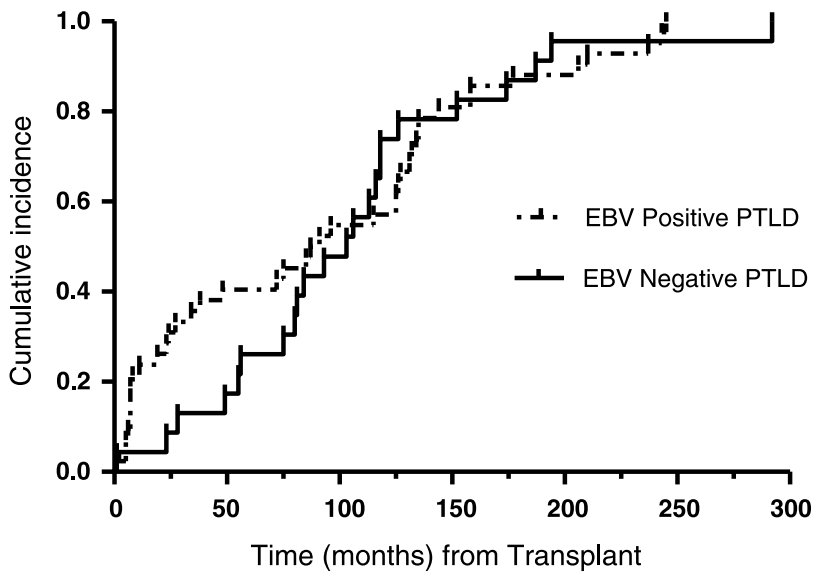

FIGURE 2. Time from transplantation (months) to PTLD diagnosis. A, Kaplan-Meier time to event plot for EBV-seropositive and EBV-seronegative recipients. The median time to presentation of PTLD was significantly shorter in EBV-seronegative recipients ( $P=0.003$, Mann-Whitney). B, Kaplan-Meier time to event plot for patients with EBV-positive PTLD and EBVnegative PTLD (89 vs. 109 months, respectively; ns, Mann-Whitney). EBV, Epstein-Barr virus; PTLD, posttransplantation lymphoproliferative disorder.

period than in the first 4 years. The etiology of late PTLD appears complex and the incidence of late disease may increase over the next 10 years as transplantation rates increase and improved graft survival results in the exposure of recipients to immunomodulatory medication for longer periods of time. Late disease may relate to impaired immune responses at the time of primary infection, duration of exposure to immunosuppression, age, immunosenescence, and perhaps other infective agents. Cytomegalovirus infection has been associated with EBV-negative PTLD (12).

Currently, the UK (2011) (19) and Kidney Disease: Improving Global Outcomes (2009) (20) guidelines advocate that high-risk EBV donor-positive, recipient-negative patients have EBV viral load monitoring until the end of the first year and that total immunosuppression is reduced when EBV titers rise significantly. However, in the United Kingdom, detection of whole-blood EBV DNA by polymerase chain reaction in the first 90 days after transplantation was reported in $50 \%$ of seronegative recipients and $56 \%$ of all adult renal transplant recipients, without the presentation of PTLD during a 2-year follow-up (21). In 20 EBV donor-positive, recipient-negative transplants receiving ATG induction, Martin et al. (22) reported detectable EBV DNA in plasma in $74 \%$ of patients in the first year, of which 1 of 20 developed PTLD. Screening for detectable EBV viral loads beyond the first year is not yet standard practice. Detection of EBV DNA in whole blood is reported in up to $36 \%$ of stable renal transplant recipients at any one time and the prevalence of DNAemia may increase with time from transplantation (23). Further, $15 \%$ of all our late cases of PTLD had no detectable EBV DNA in their blood at the time of diagnosis. Whole-blood screening for EBV DNA is sensible and logistically feasible in seronegative recipients in the first year, but viral load detection alone does not identify PTLD cases. Close clinical surveillance is also required in the high-risk patient for the duration of their transplant life. In our series, we identified clinical features that may help to prompt urgent investigations. Common symptoms included neck pain and swelling, oropharyngeal complaints, abdominal pain, obstructive symptoms, and B-symptoms. Disease presenting early and in the seronegative patient often involved multiple sites, extranodal sites, liver, spleen, and the gastrointestinal tract. Disease involving the CNS, skin, and soft tissue tended to occur late. Very late disease ( $>10$ years) may have a high rate of relapse and involve neck, oropharynx, and sites of chronic inflammation.

\section{Limitations of the Study}

EBV serostatus was missing for $32 \%$ of the cases and EBV tissue status for $30 \%$ of the cases. Data could not be collected if absent from medical records and where stored serum or histology blocks were not available for analysis. PTLD presentations after 2000 had more complete data sets: serostatus $84 \%$ (after 2000) versus $47 \%$ (before 2000) and tissue status $86 \%$ versus $47 \%$. We have no evidence to suggest any bias attached to the missing data.

Individuals whose first presentation of PTLD followed the onset of graft failure and return to dialysis were not included in the study. Although most wean or discontinue immunosuppression, a number remain on low-level immunosuppression and retain their graft, a potential site of chronic inflammation, and may continue to have an increased risk of lymphoma development. Comparison with rates of lymphoma in dialysis patients and individuals with failed kidney transplants on dialysis would be interesting.

Patients aged 18 or less years at the time of PTLD diagnosis and/or the time of transplantation were also excluded from this study. Data and characteristics of late presenting PTLD in the paediatric group are also lacking. 
Statistical analysis of differences in characteristics between patient subgroups (Table 3) is limited by the small numbers of cases. This is reflected in the wide 95\% CIs seen, for example, in comparisons between early- and lateonset disease.

\section{IMATERIALS AND METHODS}

\section{Identification of Posttransplantation Lymphoproliferative Disorder Cases}

Inclusion criteria for the study were age more than 18 years and a functioning renal allograft at the time of diagnosis. All PTLD cases were classified according to the 2008 World Health Organization classification as early, polymorphic, monomorphic, or Hodgkin's disease (24). Multiple myeloma was not included. PTLD histologic classification and subclassification and PTLD tumor EBV status based on immunohistochemical staining or in situ hybridization for EBV-encoded RNA were performed by regional specialist hematopathologists.

Data were abstracted from the Regional Tissue Typing Laboratory database and through a comprehensive search of pathology databases. Cases of PTLD occurring from August 1, 2009 onward were identified prospectively following referral to regional hemato-oncology. In the case of individuals who transferred their care to other units outside our region following transplantation $(n=175)$, outcome data including occurrence of graft loss (23 of 175) and PTLD $(n=4)$ was obtained from the National Health Service Blood and Transplant Registry. PTLD cases identified were verified with the unit providing follow-up care.

\section{Incidence of Posttransplantation Lymphoproliferative Disorder}

Patient-years of follow-up were calculated for all individuals receiving a kidney transplant between March 22, 1968 and February 29, 2012. Censorship occurred at the date of disease onset for PTLD cases and for all others at the onset of graft failure or death or the end of the follow-up period. Incidence rates were expressed as the number of cases over the number of patient-years at risk and then as cases per 1000 patient-years. Poisson distribution with 95\% CIs were calculated for the estimated rates.

We calculated the incidence of PTLD in our adult transplant population and compared with age-standardized rates of Hodgkin's lymphoma and NHL in the general population (ages $>19$ years) in England (total population $\mathrm{n}=39,104,000)$ for the calendar year 2008. Lymphoma incidence in the general population was obtained for the International Classification of Disease, Tenth Edition code C81 (Hodgkin's lymphoma) and codes C82-85 and 96 (NHL) from the North West Cancer Intelligence Service (personal communication) and UK Cancer Intelligence Service report (2011) for cases occurring in 2008 and registered by September 2010 (16).

Change in incidence rates of PTLD with increasing time from transplantation and between different eras of immunosuppression was analyzed, including 1990 to 1994 (cyclosporine, azathioprine, and prednisolone), 1995 to 1999 (cyclosporine, mycophenolate, and prednisolone), 2000 to 2004 (tacrolimus, mycophenolate and prednisolone), and since 2004 in line with National Institute for Clinical Excellence guidance (basiliximab induction followed by calcineurin inhibition with tacrolimus and added mycophenolate, azathioprine, and corticosteroids depending on immunologic risk) (25). Lymphocyte-depleting antibodies are not used as induction agents in our unit and are reserved for steroid-resistant and vascular rejection. Acute rejection rates with current immunosuppressive protocols are less than $10 \%$ and the use of ATG has decreased in recent times. Antiviral prophylaxis is given to individuals at high risk of cytomegalovirus disease (donor-positive, recipient-negative and those receiving lymphocyte-depleting antibodies).

\section{Clinical Presentation}

Groups were compared using mean (standard deviation) for normally distributed variables and median (IQR) for skewed data. Group comparisons between early- and late-onset presentations and disease presenting in EBVseronegative and EBV-seropositive recipients used chi-square testing for categorical variables and Student's two-sided $t$ test for continuous variables.
Group comparisons and univariate analyses were performed using GraphPad Prism 5 software.

\section{Epstein-Barr Virus Serostatus and Timing of Posttransplantation Lymphoproliferative Disorder}

Where EBV serostatus was not known (pre-2008 donors and recipients were not routinely screened for EBV serostatus), stored $\left(-80^{\circ} \mathrm{C}\right)$ serum samples taken on the day of transplantation were obtained from the Regional Tissue Typing Laboratory, and IgG antibody status to EBV VCA and EBNA-1 antigens was determined.

Kaplan-Meier time to event curves were used to graphically illustrate the cumulative time to presentation for EBV-seronegative and EBV-seropositive recipients (Fig. 2A) and tissue EBV-positive and tissue EBV-negative recipients (Fig. 2B). Comparison of the curves used the log-rank test (GraphPad Prism 5 software).

\section{Ethical Approval}

Regional Ethical Committee approvals for the study (Greater Manchester NWREC II/NW/0001 and NEREC 09/H1013/71) and honorary contracts were obtained. Informed consent was obtained from all living subjects.

\section{ACKNOWLEDGIMENTS}

The authors thank Drs. Susan Martin and Judith Worthington for assistance in obtaining stored serum samples from the Regional Tissue Typing Laboratory; Victoria Bowman for update and provision of the transplant database at MRI; Sabina Khan at the North West Cancer Intelligence Service, Will Hulme at National Health Service Blood and Transplant, and Stephanie Johnson and Kate Atkinson at MRI for providing research support; and Professor Philip Kalra at Salford Royal Foundation Trust and Dr. Laurie Solomon at Royal Preston Hospital and their staff for assistance in reviewing cases of PTLD in recipients returned to their base hospitals.

\section{REFERENCES}

1. Agraharkar ML, Cinclair RD, Kuo YF, et al. Risk of malignancy with long-term immunosuppression in renal transplant recipients. Kidney Int 2004; 66: 383.

2. Opelz G, Dohler B. Lymphomas after solid organ transplantation: a collaborative transplant study report. Am J Transplant 2004; 4: 222.

3. Caillard S, Lelong C, Pessione F, et al. Post-transplant lymphoproliferative disorders occurring after renal transplantation in adults: report of 230 cases from the French registry. Am J Transplant 2006; 6: 2735.

4. Faull RJ, Hollett P, McDonald SP. Lymphoproliferative disease after renal transplantation in Australia and New Zealand. Transplantation 2005; 80: 193.

5. Libertiny G, Watson CJ, Gray DW, et al. Rising incidence of posttransplant lymphoproliferative disease in kidney transplant recipients. Br J Surg 2001; 88: 1330.

6. Fernberg P, Edgren G, Adami J, et al. Time trends in risk and risk determinants of non-Hodgkin lymphoma in solid organ transplant recipients. Am J Transplant 2011; 11: 2472

7. Guppy AE, Rawlings E, Madrigal JA, et al. A quantitative assay for Epstein-Barr virus-specific immunity shows interferon-gamma producing $\mathrm{CD}^{+} \mathrm{T}$ cells increase during immunosuppression reduction to treat posttransplant lymphoproliferative disease. Transplantation 2007; 84: 1534.

8. Macedo C, Donnenberg A, Popescu I, et al. EBV-specific memory $\mathrm{CD}^{+} \mathrm{T}$ cell phenotype and function in stable solid organ transplant patients. Transpl Immunol 2005; 14: 109.

9. Smets F, Latinne D, Bazin H, et al. Ratio between Epstein-Barr viral load and anti-Epstein-Barr virus specific T-cell response as a predictive marker of posttransplant lymphoproliferative disease. Transplantation 2002; 73: 1603 .

10. Dolcetti R. B lymphocytes and Epstein-Barr virus: the lesson of posttransplant lymphoproliferative disorders. Autoimmun Rev 2007; 7: 96. 
11. Capello D, Cerri M, Muti G, et al. Molecular histogenesis of post transplantation lymphoproliferative disorders. Blood 2003; 102: 3775.

12. Caillard S, Lamy FX, Quelen C, et al. Epidemiology of post transplant lymphoproliferative disorders in adult kidney and kidney pancreas recipients: report of the French registry and analysis of subgroups of lymphomas. Am J Transplant 2012; 12: 682.

13. Opelz G, Daniel V, Naujokat C, et al. Epidemiology of pretransplant EBV and CMV serostatus in relation to posttransplant non-Hodgkin lymphoma. Transplantation 2009; 88: 962.

14. Dharnidharka VR, Lamb KE, Gregg JA, et al. Associations between EBV serostatus and organ transplant type in PTLD risk: an analysis of the SRTR National Registry Data in the United States. Am J Transplant 2012; 12: 976.

15. Quinlan SC, Pfeiffer RM, Morton LM, et al. Risk factors for earlyonset and late-onset post-transplant lymphoproliferative disorder in kidney recipients in the United States. Am J Hematol 2011; 86: 206.

16. ONS. Registrations of cancer diagnosed in 2008, England, Cancer statistics registrations. London, National Statistics, 2011.

17. Hummel M, Anagnostopoulos I, Dallenbach F, et al. EBV infection patterns in Hodgkin's disease and normal lymphoid tissue: expression and cellular localization of EBV gene products. Br J Haematol 1992; 82: 689.

18. Hummel M, Anagnostopoulos I, Korbjuhn P, et al. Epstein-Barr virus in B-cell non-Hodgkin's lymphomas: unexpected infection patterns and different infection incidence in low- and high-grade types. J Pathol 1995; 175: 263.

19. Baker R, Jardine A, Andrews P. Post-operative care of the kidney transplant recipient. Renal Association Clinical Guidelines, 2011. www.renal.org/guidelines.

20. Kidney Disease: Improving Global Outcomes (KDIGO) Transplant Work Group. KDIGO clinical practice guideline for the care of kidney transplant recipients. Am J Transplant 2009; 9: S1

21. Holmes MV, Caplin B, Atkinson C, et al. Prospective monitoring of Epstein-Barr virus DNA in adult renal transplant recipients during the early posttransplant period: role of mycophenolate mofetil. Transplantation 2009; 87: 852

22. Martin SI, Dodson B, Wheeler C, et al. Monitoring infection with Epstein-Barr virus among seromismatch adult renal transplant recipients. Am J Transplant 2011; 11: 1058.

23. Morton M, Coupes B, Vallely P, et al. Increasing prevalence of EBV DNAemia with time from transplant in stable adult renal transplant recipients. Am J Transplant 2011; 11: 287.

24. Swerdlow S, Campo E, Harris N, et al. WHO classification of tumors of haematopoietic and lymphoid tissues. Lyon, IARC Press, 2008, p. 343 .

25. NICE. Immunosuppressive therapy for renal transplantation in adults. Technol Appraisal 85. 2004.

\section{eTOCs and Publish Ahead-of-Print (PAP) Alerts}

Receive the latest developments in transplantation as soon as they're available. Request delivery of Transplantation's electronic Table of Contents (eTOC) and Publish Ahead-ofPrint (PAP) Alerts.

These are fast, easy and free services to all. You will receive:

- Complete Table of Contents for all new issues.

- Notice of all Publish Ahead-of-Print articles as they are posted at the Transplantation website.

For eTOC, visit www.transplantjournal.com and click on eTOC, to subscribe via email.

For PAP alerts, go to http://journals.lww.com/transplantjournal/toc/publishahead and click to subscribe via email or RSS feed. 